\title{
DNA profiling in peripheral blood, buccal swabs, hair follicles and semen from a patient following allogeneic hematopoietic stem cells transplantation
}

\author{
YA-TING LI, MING-KUN XIE and JIN WU \\ Department of Forensic Genetics, West China School of Basic Science and Forensic Medicine, \\ Sichuan University, Chengdu, Sichuan 610041, P.R. China
}

Received June 27, 2014; Accepted July 31, 2014

DOI: $10.3892 /$ br. 2014.332

\begin{abstract}
Allogeneic peripheral blood stem cells transplantation (allo-PBSCT) or allogeneic bone marrow transplantation (allo-BMT) have been widely used to treat patients exhibiting certain severe illnesses. However, previous studies have shown that the biological materials of allo-PBSCT or allo-BMT recipients may not constitute credible materials for personal identification. In the present study, four types of commonly used samples were collected from a male individual following gender-matched allo-BMT. Autosomal short tandem repeat (STR) and Y-STR markers analysis, based on polymerase chain reaction, were used to evaluate the chimerism status. The results showed that the blood sample were all donor type, the buccal swab sample were mixed chimerism, and the sperm and hair follicle samples maintained a recipient origin of $100 \%$. In conclusion, identical results were obtained by the two methods and it was confirmed that DNA extracted from hair follicles and sperm can be used as a reference for the pre-transplant genotype DNA profile of the recipient in the gender-match allo-BMT or -PBSCT.
\end{abstract}

\section{Introduction}

Allogeneic bone marrow transplantation (allo-BMT) or allogeneic peripheral blood stem cells transplantation (allo-PBSCT) are used commonly as an effective treatment for patients with various hematological malignancies and non-malignant diseases involving bone marrow elements, including severe aplastic anemia, severe combined immunodeficiency states, lymphoma, leukemia and solid tumors (1). A number of studies (2-5) have indicated that the bone marrow stem

Correspondence to: Professor Jin Wu, Department of Forensic Genetics, West China School of Basic Science and Forensic Medicine, Sichuan University, 3-17 Renmin South Road, Chengdu, Sichuan 610041, P.R. China

E-mail:wujin2028@163.com

Key words: short tandem repeat, Y chromosome markers stem cell transplantation cells have a significant differentiation capacity as they can differentiate into neural, bone, muscular, cartilage, liver, gut, alveolar, buccal, epidermal or endothelial cells. The study by Tran et al (6) confirmed that cells derived from bone marrow migrate to the cheek and differentiate into epithelial cells.

As aforementioned, biological samples from patients who have undergone successful allo-BMT or -PBSCT may not constitute appropriate materials for individual identification or paternity testing.

The aim of the present study was to exploit the sequence-specific primer-based polymerase chain reaction (PCR)-amplified short tandem repeat (STR) analysis method to verify whether blood, hair follicles, seminal stains and buccal swabs are appropriate for personal identification or paternity testing for patients who have undergone a successful allo-PBSCT or -BMT.

\section{Case report}

Patient. A 34-year-old male that underwent allo-BMT for chronic myelogenous leukemia requested a paternity test in the Department of Forensic Genetics at the Sichuan University (Sichuan, China). Due to the unique nature of the case, the patient was invited to participate in the chimerism study and informed consent was obtained. As to the current analysis phase, it was 13 years since the patient underwent a successful allo-BMT from an unrelated male donor. There was no evidence of chronic graft-versus-host disease involving the oral mucosa when sampling from the patient.

The post-transplant peripheral blood was collected in EDTA-coated tubes and stored at $-20^{\circ} \mathrm{C}$. Buccal swabs with oral epithelial cells were collected from both sides of the oral cavity. Prior to obtaining the buccal swabs, adequate mouthwash was required. The hair follicles were collected from different areas of the scalp. A seminal stain with sperm cells was collected in sterilized gauze and preserved in a dry environment. The oral and hair samples were maintained at room temperature until DNA extraction was performed. The patient provided the pre-transplant blood-stain sample.

DNA extraction. Genomic DNA from all the investigated materials was extracted using Chelex-100 (7). To optimize 
the extraction procedure, one $0.5-\mathrm{cm}^{2}$ portion was cut from the buccal swabs, the gauze containing the sperm and the blood stain, respectively. For extraction of the hair samples, a $0.5-\mathrm{cm}$ hair fragment, including the root, was cut. Blood (10 $\mu \mathrm{l})$, processed buccal swab, seminal stain, blood stain and hair follicle samples were transferred into a sterilized 200- $\mu 1$ eppendorf tube containing $70 \mu 1$ 5\% Chelex-100 and $5 \mu \mathrm{l}$ proteinase $\mathrm{K}(10 \mathrm{mg} / \mathrm{ml})$. The samples were incubated for $90 \mathrm{~min}$ at $56^{\circ} \mathrm{C}$, and subsequently heated at $98^{\circ} \mathrm{C}$ for $10 \mathrm{~min}$ and centrifuged at $13,000 \mathrm{x}$ g for $5 \mathrm{~min}$. The DNA concentration of each sample was evaluated with the NanoDrop ND-1000 Spectrophotometer (Thermo Fisher Scientific, Wilmington, DE, USA).

DNA profiling. The 22 autosomal unlinked loci and an additional gender-determining marker, amelogenin, were amplified with the reagents contained in the Expressmarker 22 STR loci direct PCR amplification kit (AGCU Biotechnology Co., Wuxi, China) and analyzed in the GeneAmp ${ }^{\circledR}$ PCR System 9700 (Applied Biosystems, Foster City, CA, USA). The PCR products were detected on an ABI PRISM ${ }^{\circledR}$ 3130 Genetic Anaylzer (Applied Biosystems) according to the manufacturer's instructions (8). The electrophoresis data were analyzed using GeneMapper IDX software (Applied Biosystems).

Profiling of the 22 STR loci along the Y-chromosome was carried out using the Prototype PowerPlex Y23 System kit (Promega Corp., Madison, WI, USA) following the manufacturer's instructions (9). Subsequently, allele detection was performed with GeneMapper ID v3.2 software (Applied Biosystems).

\section{Results and Discussion}

DNA profiling in all the samples are shown in Table I. In the study, the autosomal and Y-STR markers analyses of blood were $100 \%$ donor type. The DNA profiling obtained from the hair follicle and sperm samples were $100 \%$ recipient type. However, in the buccal swab samples, the result showed a manifest mixture of two sources of DNA (58\% donor). There was no evidence of contamination in any sample.

In forensic science, DNA material recovered from a crime scene has become standard forensic evidence for investigating and solving a wide spectrum of crimes, including murder and rape (10). Forensic scientists use DNA from various biological samples found at crime scenes to identify the criminal suspect by DNA profiling. Different types of DNA material have the ability to undergo a DNA match based on a hypothesis that all the cells in the human body have a consistent DNA profile (11).

However, in certain unique cases, this hypothesis is invalid. For any subject who has undergone a successful allo-BMT or -PBSCT for various severe diseases, blood is not suitable for personal identification due to a conversion to complete donor type (12). When blood cells become the donor genotype, it was previously believed that the other cells remain the same as the recipient origin. However, previous studies (2-5) indicate that stem cells can differentiate into a number of cell types in vivo. A previous study identified that male bone marrow-derived cells differentiate into
Table I. Results of the STR profiling.

\begin{tabular}{|c|c|c|c|c|c|}
\hline STR loci & $\begin{array}{c}\text { Buccal } \\
\text { swab }\end{array}$ & Blood & Hair & Sperm & $\begin{array}{c}\text { Pre- } \\
\text { transplant }\end{array}$ \\
\hline \multicolumn{6}{|l|}{ Autosomal } \\
\hline D3S1358 & $15,16,17$ & 15 & 16,17 & 16,17 & 16,17 \\
\hline D13S317 & 11,12 & 11,12 & 11,12 & 11,12 & 11,12 \\
\hline D7S820 & 10,11 & 11 & 10,11 & 10,11 & 10,11 \\
\hline D16S539 & $9,11,12$ & 11,12 & 9 & 9 & 9 \\
\hline Penta E & $12,17,20$ & 17,20 & 12,20 & 12,20 & 12,20 \\
\hline D2S441 & $11,11.3$ & 11 & 11.3 & 11.3 & 11.3 \\
\hline TPOX & 8,9 & 8,9 & 8,9 & 8,9 & 8,9 \\
\hline TH01 & $7,9.3$ & 7 & $7,9.3$ & $7,9.3$ & $7,9.3$ \\
\hline D2S1338 & $17,19,20$ & 17,19 & 19,20 & 19,20 & 19,20 \\
\hline CSF1PO & 11,12 & 11,12 & 12 & 12 & 12 \\
\hline Penta D & 9,12 & 9,12 & 9 & 9 & 9 \\
\hline D10S1248 & 14,15 & 14,15 & 15 & 15 & 15 \\
\hline D19S433 & $13,14,15.2$ & 14 & $13,15.2$ & $13,15.2$ & $13,15.2$ \\
\hline Vwa & 14,19 & 14 & 19 & 19 & 19 \\
\hline D21S11 & $32,32.2,33$ & 32,33 & 32.2 & 32.2 & 32.2 \\
\hline D18S51 & $14,15,16$ & 15,16 & 14,15 & 14,15 & 14,15 \\
\hline D6S1043 & $12,13,17,19$ & 12,19 & 13,17 & 13,17 & 13,17 \\
\hline D8S1179 & $11,13,17$ & 13,17 & 11,13 & 11,13 & 11,13 \\
\hline D5S818 & $9,10,11,13$ & 10,11 & 9,13 & 9,13 & 9,13 \\
\hline D12S391 & $17,18,19,24$ & 18,24 & 17,19 & 17,19 & 17,19 \\
\hline FGA & $22,23,25$ & 23,25 & 22 & 22 & 22 \\
\hline Amelogenin & $\mathrm{X}, \mathrm{Y}$ & $\mathrm{X}, \mathrm{Y}$ & $\mathrm{X}, \mathrm{Y}$ & $\mathrm{X}, \mathrm{Y}$ & $\mathrm{X}, \mathrm{Y}$ \\
\hline \multicolumn{6}{|l|}{$\mathrm{Y}$} \\
\hline DYS576 & 17,23 & 17 & 23 & 23 & 23 \\
\hline DYS389I & 13,14 & 14 & 13 & 13 & 13 \\
\hline DYS448 & 17,20 & 17 & 20 & 20 & 20 \\
\hline DYS389 II & 29 & 29 & 29 & 29 & 29 \\
\hline DYS19 & 14,15 & 15 & 14 & 14 & 14 \\
\hline DYS391 & 10 & 10 & 10 & 10 & 10 \\
\hline DYS481 & 23,25 & 25 & 23 & 23 & 23 \\
\hline DYS549 & 12 & 12 & 12 & 12 & 12 \\
\hline DYS533 & 11 & 11 & 11 & 11 & 11 \\
\hline DYS438 & 10,11 & 10 & 11 & 11 & 11 \\
\hline DYS437 & 14,15 & 14 & 15 & 15 & 15 \\
\hline DYS570 & 18 & 18 & 18 & 18 & 18 \\
\hline DYS635 & 20,21 & 21 & 20 & 20 & 20 \\
\hline DYS390 & 24 & 24 & 24 & 24 & 24 \\
\hline DYS439 & 12,13 & 12 & 13 & 13 & 13 \\
\hline DYS392 & 13,14 & 13 & 14 & 14 & 14 \\
\hline DYS643 & 10,11 & 11 & 10 & 10 & 10 \\
\hline DYS393 & 12,14 & 14 & 12 & 12 & 12 \\
\hline DYS458 & 15,17 & 15 & 17 & 17 & 17 \\
\hline DYS385 & $12,13,18$ & 12,18 & 13,18 & 13,18 & 13,18 \\
\hline DYS456 & 15,17 & 17 & 15 & 15 & 15 \\
\hline YGATAH4 & 12 & 12 & 12 & 12 & 12 \\
\hline
\end{tabular}

STR, short tandem repeat.

epithelial cells and migrate into the cheek (6). Coincidentally, certain studies have detected donor-derived cells in buccal swabs $(5,12,13)$. In a study of the most frequently used 


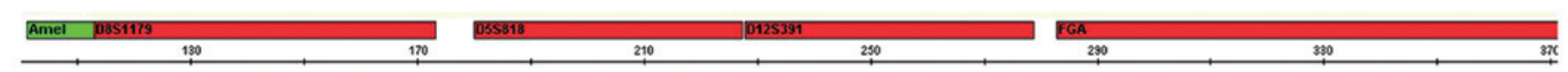

buccal swab
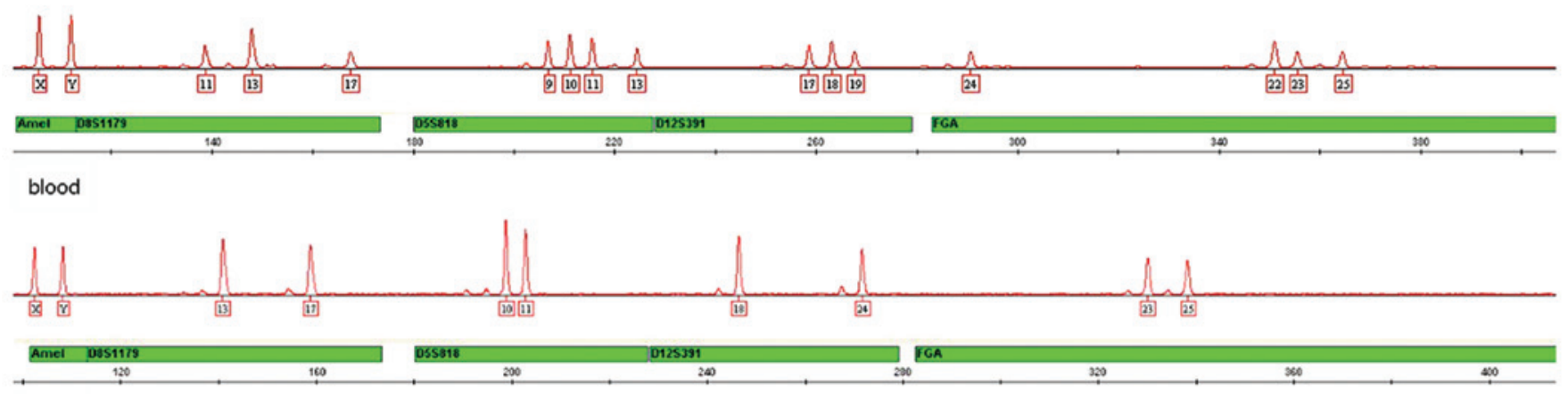

hair
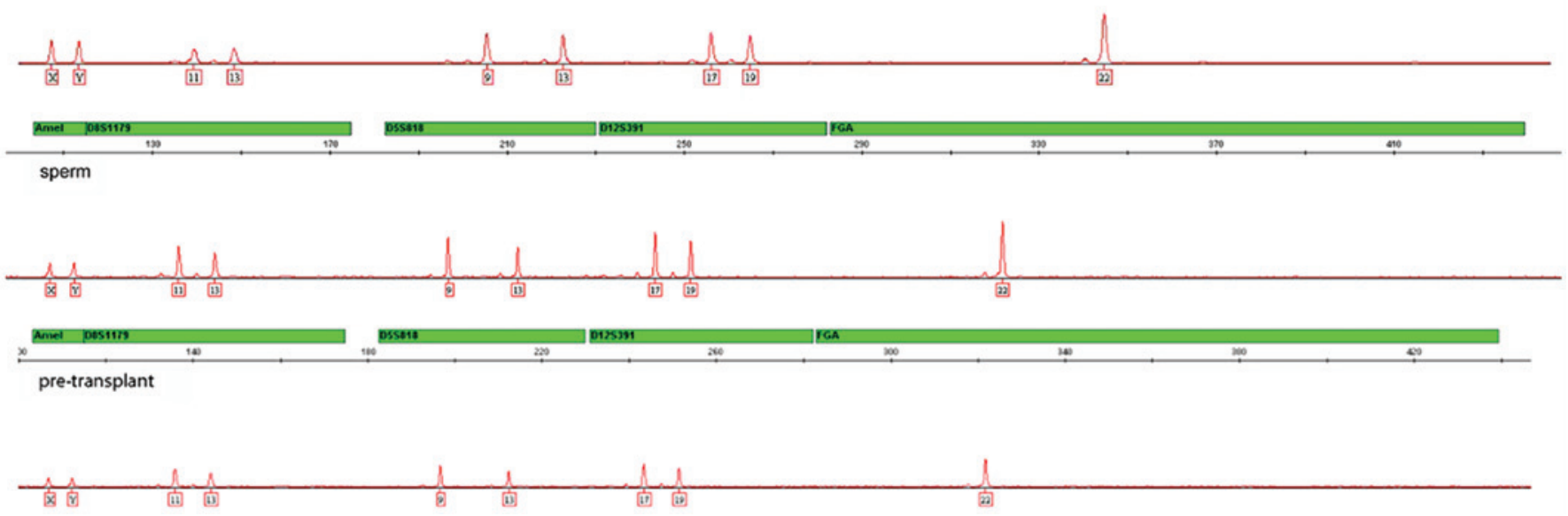

Figure 1. Representative results of autosomal STR analysis from all the samples (ROX dye-labeled markers). STR, short tandem repeat.

materials in forensic investigations, the donor-derived cells were detected not only in blood and buccal swabs, but also in fingernails (14). As with fingernails, the hair has the same ectodermal origin. However, whether the hair follicles are the only reliable source of biological material remains disputable. Semen is often used as critical evidence in forensic cases, and determining whether the genotype of semen is changed is essential. Mammalian spermatogenesis is a complicated progress; a mature spermatozoa is differentiated from the spermatogonial stem cells (15). There is no evidence that bone marrow stem cells are able to differentiate into sperm cells.

Regarding this, autosomal STR-PCR analysis is frequently applied to evaluate the origin of cells following allo-BMT or-PBSCT. In the present study, the analysis of autosomal STR markers was performed in the origin materials from the recipient using the Expressmarker 22 STR loci direct PCR amplification kit. The Expressmarker 22 system is a PCR-based amplification kit that targets a panel of 21 autosomal unlinked STR loci, which are D3S1358, D13S317, D7S820, D16S539, Penta E, D2S441 (FAM dye-labeled), TPOX, TH01, D2S1338, CSF1PO, Penta D, D10S1248 (HEX dye-labeled), D19S433, vWA, D21S11, D18S51, D6S1043 (TAMRA dye-labeled), D8S1179, D5S818, D12S391 and FGA (ROX dye-labeled), and an additional gender-determining marker Amelogenin (ROX dye-labeled), which was used to distinguish the $\mathrm{X}$ and $\mathrm{Y}$ chromosomes. These autosomal STR analysis results are in accordance with earlier study (12), as the hair follicles of the recipient following successful allo-BMT had a completely autologous profile. The genotypes that were detected in the blood samples were derived entirely from the donor, and the genotypes detected in the buccal swab samples were mixed chimerism. The results of autosomal STR analysis from the sperm sample were identical to the results from the hair follicles. These observations indicate that the DNA profiling detected from the hair follicle and sperm samples were $100 \%$ recipient type. In the buccal swab samples, the results showed two apparent sources of DNA (the mixture of the recipient and donor patterns). According to the standardized calculation procedure of chimerism values (16), the peak area from the donor accounted for $58 \%$ of the total signal. The results are presented in Fig. 1 (ROX dye-labeled markers).

From the autosomal STR markers analysis, the results supported the previous conclusion that considered hair follicle and sperm as the only reliable source of biological material for forensic personal identification. In the present study, the male patient received HLA-matched transplantation from an unrelated male donor 13 years ago. Based on the gender-matched engraftment, Y-STR multiplex analysis was performed using 


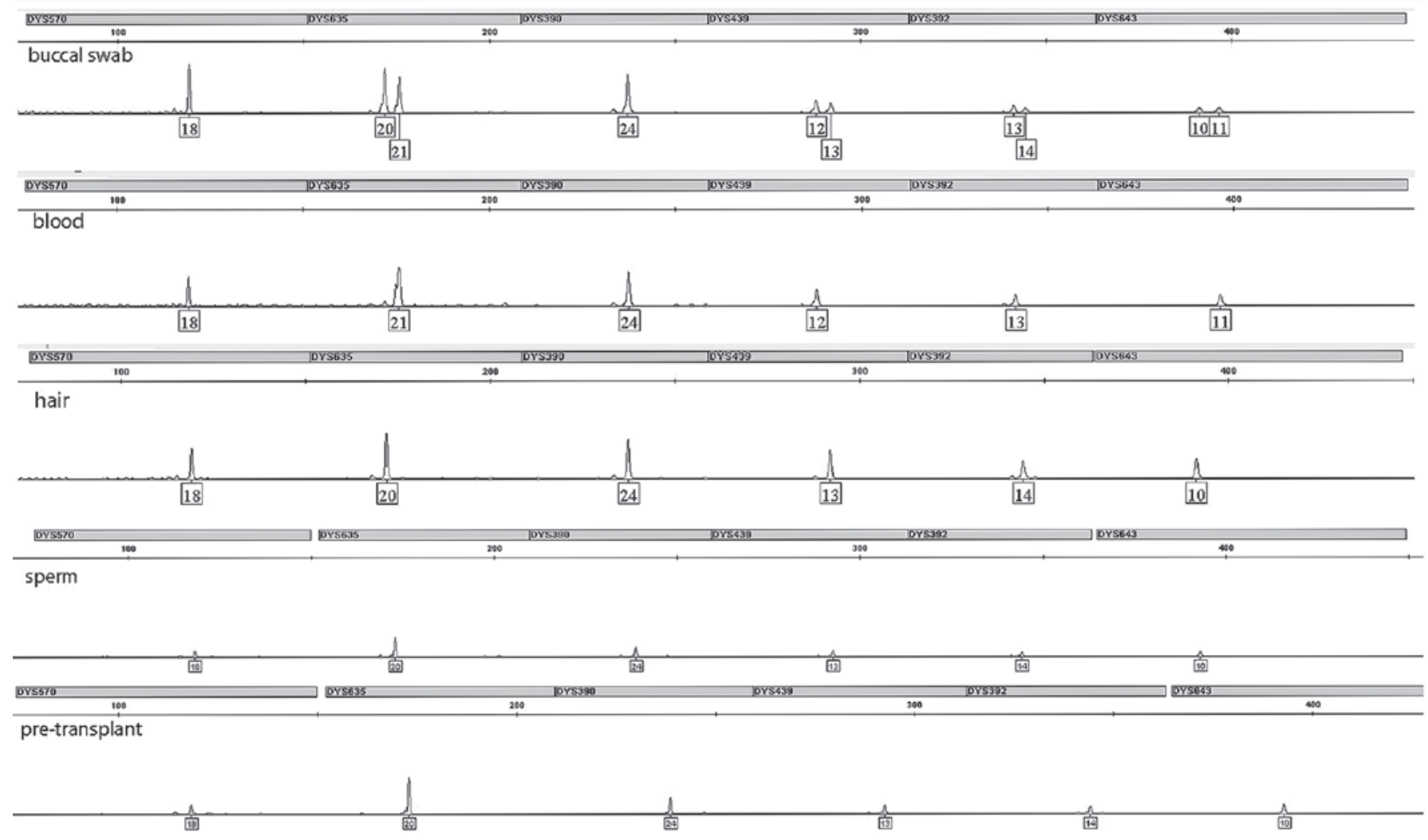

Figure 2. Representative results of Y-STR analysis from all the samples (electrophoretic profiles of DYS570, DYS635, DYS390, DYS439, DYS392 and DYS643 loci). STR, short tandem repeat.

the Prototype PowerPlex Y23 System as the subsequent step. The PowerPlex Y23 System was able to detect the minor alleles in male/male DNA admixtures (17). According to the manufacturer's instructions, all the samples were obtained and demonstrated successful typing. Fig. 2 shows the representative electrophoretic profiles of the DYS570, DYS635, DYS390, DYS439, DYS392 and DYS643 loci. The profiles indicated a mixture of the recipient and donor patterns in the buccal swab, the full donor pattern in the blood sample, and the full recipient profile in the hair follicle and sperm samples. However, previous studies revealed the Y-STR haplotype from the donor in all the post-transplant blood and buccal swab samples, and even in post-transplant hair follicle samples obtained from female patients following gender-mismatched engraftment $(11,13)$. A previous study has already demonstrated that an extremely small quantity of male material in mixed male/female DNA samples can be detected by analysis with the Y-STR system (18). The main reason behind this is due to the lack of Y-STR haplotype in female DNA, which may not affect the specificity of the Y-STR system. Notably however, the presence of the minor contribution of Y-STRs may not be revealed when using the identical Y-STR system in male/male admixture conditions.

In the present study, the results have to be considered following the DNA analysis of samples, which are the most commonly used sources for forensic and clinical genetic investigations. As adult stem cells differentiate into blood and epithelial cells, buccal swab may not serve as an ideal source of pre-transplant DNA profiling of the recipient origin. The genotypes that were detected from blood samples were completely derived from the donor. However, due to the highly-conserved process of spermatogenesis, as shown in the results, the DNA typing analysis from the sperm sample represents the recipient genotype prior to transplantation. Regarding the hair follicle sample, it has been shown that the DNA profiling remains completely the same as the recipient origin.

In conclusion, the analysis based on autosomal and Y-chromosome STR markers, which was performed in a male recipient with gender-matched allo-BMT, showed that identical results were obtained using the two methods. The investigation confirmed that the DNA extracted from hair follicles and sperm can be used as a reference for the pre-transplant genotype DNA profile of the recipient in the gender-match allo-BMT or -PBSCT. However, further studies should be conducted to verify these findings in larger samples.

\section{Acknowledgements}

The present study was financially supported by the Sichuan Province Science and Technology Support Project (grant no. 2014SZ0012).

\section{References}

1. Hardy RE and Ikpeazu EV: Bone marrow transplantation: a review. J Natl Med Assoc 81: 518-523, 1989.

2. Theise ND, Badve S, Saxena R, et al: Derivation of hepatocytes from bone marrow cells in mice after radiation-induced myeloablation. Hepatology 31: 235-240, 2000. 
3. Krause DS, Theise ND, Collector MI, et al: Multi-organ, multi-lineage engraftment by a single bone marrow-derived stem cell. Cell 105: 369-377, 2001.

4. Mezey E, Chandross KJ, Harta G, Maki RA and McKercher SR: Turning blood into brain: cells bearing neuronal antigens generated in vivo from bone marrow. Science 290: 1779-1782, 2000.

5. Zhou Y, Li S, Zhou J, et al: DNA profiling in blood, buccal swabs and hair follicles of patients after allogeneic peripheral blood stem cells transplantation. Leg Med (Tokyo) 13: 47-51, 2011.

6. Tran SD, Pillemer SR, Dutra A, et al: Differentiation of human bone marrow-derived cells into buccal epithelial cells in vivo: a molecular analytical study. Lancet 361: 1084-1088, 2003.

7. Walsh PS, Metzger DA and Higushi R: Chelex 100 as a medium for simple extraction of DNA for PCR-based typing from forensic material. BioTechniques 10(4): 506-13 (April 1991). Biotechniques 54: 134-139, 2013.

8. Zou KN, Cao Y, Xia ZF, Zheng WG and Zhou HG: Forensic application of expressmarker 22 STR loci direct PCR amplification kit. Fa Yi Xue Za Zhi 28: 448-450, 2012 (In Chinese).

9. Davis C, Ge J, Sprecher C, et al: Prototype PowerPlex ${ }^{\circledR}$ Y 23 System: a concordance study. Forensic Sci Int Genet 7: 204-208, 2013.

10. Bond JW and Hammond C: The value of DNA material recovered from crime scenes. J Forensic Sci 53: 797-801, 2008.

11. Jacewicz R, Lewandowski K, Rupa-Matysek J, Jedrzejczyk M, Komarnicki M and Berent J: Genetic investigation of biological materials from patients after stem cell transplantation based on autosomal as well as Y-chromosomal markers. Int J Legal Med 127: 359-362, 2013.
12. Hong YC, Liu HM, Chen PS, et al: Hair follicle: a reliable source of recipient origin after allogeneic hematopoietic stem cell transplantation. Bone Marrow Transplant 40: 871-874, 2007.

13. Jacewicz R, Lewandowski K, Rupa-Matysek J, et al: Donor-derived DNA in hair follicles of recipients after allogeneic hematopoietic stem cell transplantation. Bone Marrow Transplant 45: 1638-1644, 2010.

14. Imanishi D, Miyazaki Y, Yamasaki R, et al: Donor-derived DNA in fingernails among recipients of allogeneic hematopoietic stem-cell transplants. Blood 110: 2231-2234, 2007.

15. Brinster RL and Zimmermann JW: Spermatogenesis following male germ-cell transplantation. Proc Natl Acad Sci USA 91: 11298-11302, 1994.

16. Nollet F, Billiet J, Selleslag D and Criel A: Standardisation of multiplex fluorescent short tandem repeat analysis for chimerism testing. Bone Marrow Transplant 28: 511-518, 2001.

17. Mayntz-Press KA and Ballantyne J: Performance characteristics of commercial Y-STR multiplex systems. J Forensic Sci 52: 1025-1034, 2007.

18. Cerri N, Ricci U, Sani I, Verzeletti A and De Ferrari F: Mixed stains from sexual assault cases: autosomal or Y-chromosome short tandem repeats? Croat Med J 44: 289-292, 2003. 